\title{
Equitable matroids
}

\author{
Dillon Mayhew* \\ Mathematical Institute \\ University of Oxford \\ 24-29 St Giles \\ Oxford, OX1 3LB \\ United Kingdom
}

Submitted: Apr 3, 2006; Accepted: Apr 19, 2006; Published: Apr 24, 2006

Mathematics Subject Classification: 05B35, 05A05

\begin{abstract}
One way to choose a basis of a matroid at random is to choose an ordering of the ground set uniformly at random and then use the greedy algorithm to find a basis. We investigate the class of matroids having the property that this procedure yields a basis uniformly at random. We show how this class is related to some other naturally-defined families of matroids and consider how it behaves under well-known matroid operations.
\end{abstract}

\section{Introduction}

Counting the bases of a matroid is a well-studied problem with many applications. In some cases, counting the bases exactly is known to be a computationally intractable problem $[3,7]$. Therefore a considerable amount of attention has been paid to producing approximations to the number of bases $[1,2]$. Work by Jerrum, Valiant, and Vazirani [8] shows that this task is intimately connected to the problem of choosing a basis uniformly at random.

Because of this connection between counting bases and choosing them uniformly at random, a great deal of effort has been spent in the search for efficient ways to randomly select a basis of a matroid [6,11]. Perhaps the most obvious way to choose a basis of a matroid at random is to use an implementation of the greedy algorithm. Let $M$ be a matroid on the ground set $E$ and let $\rho$ be a linear order on $E$. Then $\rho$ induces a natural lexicographical order on the subsets of $E$. The greedy algorithm finds the basis that is minimum in this lexicographical order.

${ }^{*}$ Current address: School of Mathematics, Statistics and Computer Science, Victoria University of Wellington, PO BOX 600, Wellington, New Zealand. Email: dillon.mayhew@mcs.vuw.ac.nz 
In general though, if $\rho$ is chosen uniformly at random from all linear orderings of $E$, some bases of $M$ have a greater chance than others of being the output of this algorithm. In this paper we investigate the class of matroids such that if $\rho$ is chosen uniformly at random, then the output of the greedy algorithm is uniformly distributed over the bases of the matroid.

Let $M$ be a matroid on a ground set $E$ and suppose that $\rho$ is a linear order on $E$. Let $B(\rho)$ be the minimum basis in the lexicographical order induced by $\rho$. Suppose that $B$ is a basis of $M$. Define $p(B, M)$ to be the probability that $B(\rho)=B$, given that $\rho$ is chosen uniformly at random from the set of linear orders of $E$.

Definition 1.1. Let $M$ be a matroid. If $p(B, M)=p\left(B^{\prime}, M\right)$ for any two bases $B$ and $B^{\prime}$ of $M$, then $M$ is equitable.

Let $O(B, M)$ be the number of linear orders $\rho$ of $E$ such that $B=B(\rho)$. Clearly $p(B, M)=O(B, M) / n$ !, where $n=|E|$, so we may equivalently say that $M$ is equitable if $O(B, M)=O\left(B^{\prime}, M\right)$ for any two bases $B$ and $B^{\prime}$ of $M$.

In this article we describe some properties of equitable matroids and consider their behaviour under some basic matroid operations. We note that a few previously-studied classes of matroids, such as perfect matroid designs and basis-transitive matroids, are contained in the set of equitable matroids. Like those two classes, the class of equitable matroids contains some well-known families of matroids as well as several sporadic members, a few of which we exhibit. We refer to Oxley [10] for the basic concepts of matroid theory. Terminology and notation will follow that source.

\section{Basic properties of equitable matroids}

If $M$ is a uniform matroid, then $O(B, M)=r(M) ! r\left(M^{*}\right)$ ! for any basis $B$, so the next result follows.

Proposition 2.1. All uniform matroids are equitable.

A simple necessary condition for a matroid to be equitable follows almost immediately from the definition.

Proposition 2.2. The number of bases of an equitable matroid on n elements divides n!.

The necessary condition in Proposition 2.2 is not sufficient:

Example 1. Let $M$ be the rank-2 matroid on the ground set $\{1, \ldots, 6\}$ where the only non-trivial parallel class of $M$ is $\{3,4,5,6\}$. Then the number of bases of $M$ is 9 , which divides 6 !. However, if $B_{1}$ is the basis $\{1,2\}$ and $B_{2}$ is the basis $\{1,3\}$, then it is easy to confirm that $O\left(B_{1}, M\right)=48$ while $O\left(B_{2}, M\right)=84$.

Let $M$ be a matroid on the ground set $E$ and let $B$ be a basis of $M$. If $e \in E-B$, then the unique circuit in $B \cup e$ is denoted by $C(e, B)$. Let the partial order $\tau(B, M)$ on $E$ be defined so that $x \leqslant_{\tau(B, M)} y$ if and only if (i) $x=y$ or (ii) $x \in B, y \in E-B$, and $x \in C(y, B)$. A linear order $\rho$ of $E$ is a linear extension of $\tau(B, M)$ if $x \leqslant_{\tau(B, M)} y$ implies $x \leqslant \rho y$. The next result is not difficult. 
Proposition 2.3. Let $M$ be a matroid on $E$, and let $B$ be a basis of $M$. Suppose $\rho$ is a linear order of $E$. Then $B(\rho)=B$ if and only if $\rho$ is a linear extension of $\tau(B, M)$.

It is elementary to demonstrate that $x \leqslant_{\tau(B, M)} y$ if and only if $y \leqslant_{\tau\left(E-B, M^{*}\right)} x$. The next proposition follows immediately from this fact and from Proposition 2.3.

Proposition 2.4. If $M$ is equitable, then so is $M^{*}$.

Suppose that $\left(X_{1}, X_{2}\right)$ is a separation of a matroid $M$. Let $x_{i}=\left|X_{i}\right|$ and let $\rho_{i}$ be a linear order of $X_{i}$ for $i=1,2$. There are $\left(\begin{array}{c}x_{1}+x_{2} \\ x_{1}\end{array}\right)$ linear orders $\rho$ of $E(M)$ such that, for all $i \in\{1,2\}$ and all $x, y \in X_{i}, x \leqslant_{\rho_{i}} y$ implies $x \leqslant \rho$. This, combined with the fact that the basis $B$ of $M$ is minimum in the lexicographic order induced by $\rho$ if and only if $B \cap X_{i}$ is a minimum basis of $M \mid X_{i}$ for all $i \in\{1,2\}$, yields the fact that

$$
O(B, M)=\left(\begin{array}{c}
x_{1}+x_{2} \\
x_{1}
\end{array}\right) O\left(B \cap X_{1}, M \mid X_{1}\right) O\left(B \cap X_{2}, M \mid X_{2}\right) .
$$

The next result follows.

Proposition 2.5. A matroid is equitable if and only if all of its connected components are.

We have shown that the class of equitable matroids is closed under duality and direct sums. We shall see later that a minor of an equitable matroid need not be equitable.

\section{Super-equitable matroids}

In this section we describe a family of matroids that is properly contained in the class of equitable matroids and which contains many of the most familar equitable matroids. Let $M$ be a matroid on the ground set $E$ and let $B$ be a basis of $M$. Let $G(B, M)$ be the bipartite graph which has $E$ as its vertex set and $\{\{x, y\} \mid x \in B, y \in E-B, x \in$ $C(y, B)\}$ as its edge set. Note that $G(B, M)$ is isomorphic to the graph underlying the Hasse diagram of the partial order $\tau(B, M)$.

Definition 3.1. A matroid $M$ is super-equitable if $G(B, M)$ and $G\left(B^{\prime}, M\right)$ are isomorphic whenever $B$ and $B^{\prime}$ are bases of $M$.

Clearly every uniform matroid is super-equitable. The next result is obvious.

Proposition 3.2. If $M$ is super-equitable, then so is $M^{*}$.

We shall prove that every super-equitable matroid is equitable after some intermediary results. The first follows from [10, Lemma 10.2.8, Corollary 10.2.9].

Lemma 3.3. The vertex sets of the connected components of $G(B, M)$ are the ground sets of connected components of $M$. 
The 'if' direction of the next result is very easy. To prove the converse we note that any isomorphism between $G(B, M)$ and $G\left(B^{\prime}, M\right)$, where $B$ and $B^{\prime}$ are two bases of the matroid $M$, must take connected components of $M$ to other connected components by Lemma 3.3. Now it is not difficult to show that if $B$ and $B^{\prime}$ differ only in a single connected component $M^{\prime}$ of $M$, then there must be an isomorphism between $G\left(B \cap E\left(M^{\prime}\right), M^{\prime}\right)$ and $G\left(B^{\prime} \cap E\left(M^{\prime}\right), M^{\prime}\right)$.

Proposition 3.4. A matroid is super-equitable if and only if all of its connected components are.

Proposition 3.5. Every super-equitable matroid is equitable.

Proof. By Propositions 2.5 and 3.4, it will suffice to prove the result for connected superequitable matroids. Let us therefore suppose that $M$ is a connected super-equitable matroid on the ground set $E$. Let $B$ and $B^{\prime}$ be two bases of $M$. Since $G(B, M)$ and $G\left(B^{\prime}, M\right)$ are connected bipartite graphs, it follows that the isomorphism that takes $G(B, M)$ to $G\left(B^{\prime}, M\right)$ is either an isomorphism or an anti-isomorphism between the partial orders $\tau(B, M)$ and $\tau\left(B^{\prime}, M\right)$. In either case, the number of linear extensions of $\tau(B, M)$ must be the number of linear extensions of $\tau\left(B^{\prime}, M\right)$. Therefore $M$ is equitable.

The converse of Proposition 3.5 is not true: the class of super-equitable matroids is properly contained in the class of equitable matroids. However, examples of matroids that are equitable without being super-equitable are somewhat hard to find. One can check that the truncation of $U_{2,20} \oplus U_{1,8} \oplus U_{1,8}$ is equitable. It is easy to verify that it is not super-equitable.

\section{Basis-transitive matroids and PMDs}

In this section we discuss two important classes of super-equitable matroids.

Observe that if $B$ and $B^{\prime}$ are bases of a matroid $M$, then any automorphism of $M$ that takes $B$ to $B^{\prime}$ is also an isomorphism between $G(B, M)$ and $G\left(B^{\prime}, M\right)$.

Definition 4.1. A matroid $M$ is basis-transitive if for any two bases $B$ and $B^{\prime}$ there exists an automorphism of $M$ that takes $B$ to $B^{\prime}$.

The next result follows from our discussion above.

Proposition 4.2. Every basis-transitive matroid is super-equitable.

Basis-transitive matroids have been studied in, for example, [4] and [9]. It is easy to see that uniform matroids are basis-transitive, and it is well known that the projective and affine geometries are basis-transitive and so are their truncations.

We now describe another class of super-equitable matroids.

Definition 4.3. If $M$ is a matroid and, for $0 \leqslant i \leqslant r(M)$, all rank- $i$ flats of $M$ have the same cardinality, then $M$ is a perfect matroid design (PMD). 
A proof that every PMD is equitable can be found in [2, Proposition 3.2.2], although the terminology used is different.

Proposition 4.4. Every PMD is super-equitable.

Proof. Let $M$ be a PMD on the set $E$. For $0 \leqslant i \leqslant r(M)$ let $\alpha_{i}$ be the size of the rank- $i$ flats of $M$. If $B$ is a basis of $M$ and $X$ is a subset of $B$, then let $f_{B}(X)$ be the set $\{e \in E-B \mid C(e, B)=X \cup e\}$.

We claim that for any integer $0 \leqslant i \leqslant r(M)$ there is an integer $\beta_{i}$, such that if $B$ is any basis of $M$ and $X$ is any subset of $B$ of size $i$, then $\left|f_{B}(X)\right|=\beta_{i}$. Clearly $\beta_{0}$ is equal to the number of loops of $M$. Suppose that the claim is true when $i<k$, where $k \geqslant 1$. Let $X$ be a subset of size $k$ of a basis $B$. Note that

$$
\mathrm{cl}_{M}(X)=X \cup \bigcup_{X^{\prime} \subseteq X} f_{B}\left(X^{\prime}\right)
$$

Since $f_{B}(Y)$ and $f_{B}\left(Y^{\prime}\right)$ are disjoint if $Y \neq Y^{\prime}$, it follows from the inductive assumption that

$$
\alpha_{k}=\left|\mathrm{cl}_{M}(X)\right|=|X|+\left|f_{B}(X)\right|+\sum_{j=0}^{k-1}\left(\begin{array}{l}
k \\
j
\end{array}\right) \beta_{j} .
$$

Thus $\beta_{k}=\alpha_{k}-k-\sum_{j=0}^{k-1}\left(\begin{array}{c}k \\ j\end{array}\right) \beta_{j}$. The result follows easily.

Perfect matroid designs have been investigated in [5] and [13]. The class of PMDs includes the uniform matroids, the projective and affine geometries and their truncations. Although this indicates that the class of basis-transitive matroids has a large intersection with the class of PMDs, neither class is contained in the other, as shown by the next two examples.

Example 2. Consider a projective plane that is not a projective geometry, that is, a plane in which Desargues' Theorem does not hold. Considered as a matroid, such a plane is a PMD. However, Li's characterisation of rank-3 basis-transitive matroids [9] shows that a non-desarguesian plane cannot be basis-transitive. Therefore not every PMD is basis-transitive.

Example 3. Let $r, n$, and $t$ be integers such that $1<r<n$ and $t \geqslant 2$. Let $M$ be the truncation of the direct sum of $t$ copies of $U_{r, n}$. It is easy to show that $M$ is basis-transitive but not a PMD.

Nor is it true that every super-equitable matroid is either basis-transitive or a PMD, even if we restrict our attention to connected super-equitable matroids.

Example 4. Let $M_{8}$ be the rank-4 matroid on the ground set $A \cup B$, where $A$ and $B$ are disjoint sets of size five and three respectively, such that the only non-spanning circuits of $M$ are $B$ and any set of four elements from $A$. Thus $M_{8}$ is isomorphic to the truncation of the direct sum of $U_{3,5}$ and $U_{2,3}$. 
There are two types of bases of $M_{8}$. The first contains three elements of $A$ and one element of $B$, while the second contains two elements each from $A$ and $B$. Clearly $M_{8}$ is neither basis-transitive nor a PMD. However, it is not difficult to see that if $B_{1}$ is a basis of the first type and $B_{2}$ a basis of the second type, then both $G\left(B_{1}, M_{8}\right)$ and $G\left(B_{2}, M_{8}\right)$ are isomorphic to the graph that is produced by deleting two adjacent edges from $K_{4,4}$. Thus $M_{8}$ is super-equitable.

The next results are easy.

Proposition 4.5. If $M$ is a basis-transitive matroid then so is $M^{*}$.

Proposition 4.6. A matroid is basis-transitive if and only if all its connected components are.

In contrast, the dual of a PMD need not be a PMD [12, Section 12.6] and the class of PMDs is not closed under direct sums.

\section{Basic Operations}

We conclude by considering how the classes we have discussed behave under certain basic matroid operations.

The next example shows that the classes of basis-transitive matroids, PMDs, superequitable matroids, and equitable matroids are not closed under taking minors.

Example 5. Consider the Fano plane, $F_{7}$. We have already noted that projective geometries belong to the intersection of basis-transitive matroids and PMDs, so $F_{7}$ is superequitable, and hence equitable. However, by deleting two points from $F_{7}$ we obtain a matroid which is easily seen to be not basis-transitive, super-equitable, equitable, nor a PMD.

The truncation of a PMD is also a PMD, but the next example shows that the classes of basis-transitive matroids, super-equitable matroids, and equitable matroids are not closed under truncation.

Example 6. Let $M_{9}$ be the truncation of the direct sum of three copies of $U_{2,3}$. We noted in Example 3 that $M_{9}$ is basis-transitive, and hence super-equitable and equitable. The truncation of $M_{9}$ is a rank-4 matroid containing three disjoint non-trivial lines. It is easy to see that $T\left(M_{9}\right)$ contains two types of bases. The first type avoids one of the non-trivial lines of $T\left(M_{9}\right)$, while the second type has a non-empty intersection with each non-trivial line. It follows that $T\left(M_{9}\right)$ is not basis-transitive. Furthermore, if $B_{1}$ is a basis of the first type and $B_{2}$ a basis of the second type, then $G\left(B_{1}, M_{9}\right)$ has 16 edges and $G\left(B_{2}, M_{9}\right)$ has 18. Also, $O\left(B_{1}, M_{9}\right)=3648$, while $O\left(B_{2}, M_{9}\right)=3264$. Thus $T\left(M_{9}\right)$ is neither super-equitable nor equitable. 
It is easily seen that the free extension or free coextension of a PMD need not be a PMD. The matroid in Example 1 is the free extension of the basis-transitive matroid $U_{1,4} \oplus U_{1,1}$. Thus the classes of basis-transitive matroids, super-equitable matroids, and equitable matroids are not closed under free extensions, nor, by duality, are they closed under free coextensions.

The Higgs lift of a matroid is the restriction of the free coextension to the original ground set. The Higgs lift of the projective geometry $\mathrm{PG}(2,3)$ contains rank-3 flats of size three and four, so the class of PMDs is not closed under the Higgs lift. Nor are the classes of basis-transitive matroids, super-equitable matroids, and equitable matroids, since the matroid in Example 1 is the Higgs lift of the basis-transitive matroid $U_{0,4} \oplus U_{1,2}$.

Since the matroid in Example 1 is a paving matroid ([10, pp. 26]) and a nested matroid ([10, pp. 51]) neither of these classes are contained in the class of basis-transitive, superequitable, or equitable matroids.

\section{Acknowledgements}

I thank my supervisor, Professor Dominic Welsh, who first suggested an investigation of equitable matroids, and the referee for constructive comments.

\section{References}

[1] Y. Azar, A. Z. Broder, and A. M. Frieze. On the problem of approximating the number of bases of a matroid. Inform. Process. Lett. 50 (1994), no. 1, 9-11.

[2] L. E. Chávez Lomelí. The basis problem for matroids. MSc. Thesis, University of Oxford, 1995.

[3] C. J. Colbourn, J. S. Provan, and D. Vertigan. The complexity of computing the Tutte polynomial on transversal matroids. Combinatorica 15 (1995), no. 1, 1-10.

[4] A. Delandtsheer. Basis-homogeneous geometric lattices. J. London Math. Soc. (2) 34 (1986), no. 3, 385-393.

[5] M. Deza. Perfect matroid designs. In Matroid applications, volume 40 of Encyclopedia Math. Appl., pp. 54-72. Cambridge Univ. Press, Cambridge, 1992.

[6] T. Feder and M. Mihail. Balanced matroids. In Proceedings of the 24th Annual Association for Computing Machinery Symposium on the Theory of Computing, pp. 26-38, New York, 1992. ACM Press.

[7] O. Gimenez and M. Noy. On the complexity of computing the Tutte polynomial of bicircular matroids. Combin. Probab. Comput. 15 (2006), no. 3, 385-395.

[8] M. R. Jerrum, L. G. Valiant, and V. V. Vazirani. Random generation of combinatorial structures from a uniform distribution. Theoret. Comput. Sci. 43 (1986), no. 2-3, $169-188$. 
[9] H. L. Li. On basis-transitive geometric lattices. European J. Combin. 10 (1989), no. $6,561-573$.

[10] J. G. Oxley. Matroid theory. Oxford University Press, New York, 1992.

[11] M. Sohoni. Rapid mixing of some linear matroids and other combinatorial objects. Graphs Combin. 15 (1999), no. 1, 93-107.

[12] D. J. A. Welsh. Matroid theory. Academic Press, London, 1976.

[13] P. Young and J. Edmonds. Matroid designs. J. Res. Nat. Bur. Standards Sect. B 77B (1973), 15-44. 